\title{
Growth Cone Turning Induced by Direct Local Modification of Microtubule Dynamics
}

\author{
Kenneth B. Buck and James Q. Zheng \\ Department of Neuroscience and Cell Biology, University of Medicine and Dentistry of New Jersey, Robert Wood \\ Johnson Medical School, Piscataway, New Jersey 08854
}

Pathfinding by nerve growth cones depends on attractive and repulsive turning in response to a variety of guidance cues. Here we present direct evidence to demonstrate an essential and instructive role for microtubules (MTs) in growth cone steering. First, both growth cone attraction and repulsion induced by diffusible cues in culture can be completely blocked by low concentrations of drugs that specifically inhibit dynamic microtubule ends in the growth cone. Second, direct focal photoactivated release of the microtubule-stabilizing drug taxol on one side of the growth cone consistently induces attraction (turning toward the site of application). Using the focal pipette application method, we also show that local MT stabilization by taxol induces growth cone attraction, whereas local MT destabilization by the microtubule-disrupting drug nocodazole induces repulsion (turning away). Finally, the microtubule-initiated at-

The establishment of specific neuronal connections requires guided extension of developing axons to their appropriate targets by a variety of diffusible and surface-bound extracellular cues. The motile growth cones of elongating axons are responsible for sensing spatially and temporally distributed guidance signals that direct the growth cone to steer toward (attraction) or away from (repulsion) the guidance source to reach their targets (TessierLavigne and Goodman, 1996). It is believed that asymmetric activation of the surface receptors on the growth cone by guidance molecules elicits a cascade of localized intracellular signaling events that carry the directional signal for growth cone steering. Presumably, the code for directional steering is maintained at each level of intracellular signaling down to the cytoskeleton (Bentley and O'Connor, 1994; Jay, 1996; Zheng et al., 1996b). It has been convincingly shown that cells undergoing chemotaxis use a similar sensing mechanism (Parent and Devreotes, 1999). In nerve growth cones, actin and microtubules (MTs) are the cytoskeletal components responsible for locomotion and are the ultimate targets of directional signaling. The actin cytoskeleton is found predominately in the peripheral region (P-region) of the growth cone, where highly dynamic lamellipodia and filopodia can be found (Smith, 1988). The actin-based protrusive activity

\footnotetext{
Received May 3, 2002; revised July 19, 2002; accepted Aug. 6, 2002.

This work was supported by a grant from the National Institutes of Health. We thank Dr. Marc Tessier-Lavigne (Stanford University, Stanford, CA) for providing us the purified netrin-1 proteins and J. Gibney for her technical assistance throughout the entire project.

Correspondence should be addressed to Dr. James Zheng, Department of Neuroscience and Cell Biology, University of Medicine and Dentistry of New JerseyRobert Wood Johnson Medical School, 675 Hoes Lane, Piscataway, NJ 08854. E-mail: james.zheng@umdnj.edu.

Copyright (C) 2002 Society for Neuroscience $\quad 0270-6474 / 02 / 229358-10 \$ 15.00 / 0$
}

tractive turning requires the participation of the actin cytoskeleton: local microtubule stabilization induces preferential protrusion of lamellipodia before the attractive turning, and the attraction can be abolished by inhibition of either actin polymerization or the Rho family GTPases. Together, these results demonstrate a novel steering mechanism for growth cones in which local and selective modification of dynamic microtubules can initiate and instruct directional steering. With the subsequent concerted activity of the actin cytoskeleton, this microtubule-initiated mechanism provides the growth cone with the additional means to efficiently navigate through its environment.

Key words: growth cone; microtubule; cytoskeleton; axon guidance; actin; Rho GTPase

coupled with retrograde flow and attachment of the actin network to the membrane and substrate is thought to generate the kinetic force necessary for growth cone motility (Lin et al., 1994; Suter and Forscher, 2000). MTs are bundled together in the axonal shaft and central region (C-region) of the growth cone, whereas individual microtubules extend off the bundles and splay into the peripheral region, where they occasionally invade the actin-rich areas. These single MTs exhibit dynamic instability with characteristic rapid growth and shrinkage (Tanaka and Kirschner, 1991). Although both cytoskeletal components are fundamental for growth cone motility, it is commonly believed that the actin cytoskeleton is primarily responsible for initiating and directing growth cone steering, whereas MTs consolidate and support the new extension initiated by the preferential actin activity (Mitchison and Kirschner, 1988; Smith, 1988).

Recently, an increasing number of studies have suggested that dynamic MTs in the growth cone may play a more critical role (Tanaka and Kirschner, 1995; Tanaka et al., 1995; Challacombe et al., 1997; Mack et al., 2000) for growth cone steering. In particular, dynamic MT ends seen in the P-region of the growth cone were found to be essential for repulsive turning of the growth cone at substrate borders (Tanaka and Kirschner, 1995; Challacombe et al., 1997). Furthermore, interplay between the actin and MT cytoskeleton has been shown to be critical for axon branching (Dent and Kalil, 2001). Finally, a growing number of signaling components that can target microtubules have been identified (Gundersen and Cook, 1999). These findings raise an important question of how local MT dynamics contribute to growth cone steering. It is not clear from these studies whether microtubules play a supporting role to consolidate directional growth initiated by the actin cytoskeleton or an instructive role to 
initiate growth cone steering. In this study, we present direct evidence that dynamic MT ends in the growth cone are required for growth cone turning induced by diffusible cues, and that local modification of the dynamics of MTs is sufficient to initiate and direct the turning response.

\section{MATERIALS AND METHODS}

Cell culture. Dissociated cells from the neural tube tissue of 1-d-old Xenopus embryos (Spitzer and Lamborghini, 1976) were plated on glass coverslips precoated with Con A (Chang et al., 1998) $\left(\sim 1 \mu \mathrm{g} / \mathrm{cm}^{2} ; \mathrm{E}-\mathrm{Y}\right.$ Labs). The cultures were kept at $20-22^{\circ} \mathrm{C}$ in culture medium consisting of the following (v/v): 50\% Leibovitz L-15 medium (Invitrogen, Carlsbad, CA), $50 \%$ Ringer's solution (in mM: $115 \mathrm{KCl}, 2 \mathrm{CaCl}_{2}, 2.5 \mathrm{KCl}$, and 10 HEPES, $\mathrm{pH}$ 7.4), $1 \%$ fetal bovine serum (Invitrogen), and $50 \mathrm{ng} / \mathrm{ml}$ neurotrophin-3 (Regeneron Pharmaceuticals, Tarrytown, NY).

Growth cone turning induced by extracellular gradients. Microscopic gradients of chemicals were produced by the pipette application method described previously (Lohof et al., 1992; Zheng et al., 1996a). A standard pressure pulse of 3 psi was applied to a glass pipette (1 $\mu \mathrm{m}$ opening) at a frequency of $2 \mathrm{~Hz}$, with durations of 20 and $5 \mathrm{msec}$ for guidance molecules and MT drugs, respectively. The direction of growth cone extension at the beginning of the experiment was defined by the distal 20 $\mu \mathrm{m}$ segment of the neurite. The pipette tip was positioned $45^{\circ}$ from the initial direction of extension and $100 \mu \mathrm{m}$ away for guidance cues or 50 $\mu \mathrm{m}$ away for MT drugs from the center of the growth cone. The digital images of the growth cone at the onset and end of the $30 \mathrm{~min}$ period were acquired and overlaid with pixel-to-pixel accuracy, and the trajectory of new neurite extension was traced using Adobe PhotoShop (Adobe Systems, San Jose, CA). The turning angle was defined by the angle between the original direction of neurite extension and a line connecting the positions of the growth cone at the experiment onset and at the end of 30 min of exposure to the gradient. Neurite extension was quantified by measuring the entire trajectory of net neurite growth over the $30 \mathrm{~min}$ period. Only growth cones extending $\geq 5 \mu \mathrm{m}$ were scored for turning responses. For bath application, drugs were added to the bath medium 20 min before the onset of gradient application.

Microscopy and imaging for turning assay. All turning experiments were performed in an open chamber on an inverted Nikon (Tokyo, Japan) microscope equipped with differential interference contrast (DIC) optics and a $40 \times 1.3$ numerical aperture (NA) oil-immersion objective. A 0.5 inch CCD video camera (C2400-75i; Hamamatsu, Bridgewater, NJ) was used for video imaging in conjunction with an Argus-20 image processor (Hamamatsu) for image enhancement. The video images were background subtracted, averaged over four video frames, contrast enhanced in real time using Argus-20, and digitally acquired using a personal computer (Wang and Zheng, 1998).

Focal laser-induced photolysis of caged taxol. Focal laser-induced photolysis (FLIP) of caged taxol was achieved using the system described previously (Zheng, 2000). A nitrogen-pulsed laser (Laser Science, Franklin, MA) was focused to a spot of $\sim 2 \mu \mathrm{m}$ in diameter through a $40 \times 1.3$ NA oil-immersion objective (Nikon) for FLIP. Spatial restriction and efficiency of photoactivation were evaluated by FLIP of photosensitivecaged fluorescein dextran as described previously (Zheng, 2000). To allow the caged taxol $(1 \mu \mathrm{M})$ to reach equilibrium inside the growth cone, neurons were preincubated with caged taxol for $10 \mathrm{~min}$ before the uncaging experiments. Each growth cone was positioned such that the laser spot would be located in the P-region of the growth cone on one side and be maintained throughout the entire experiment. Images of the growth cone at various times after the onset of repetitive FLIP were digitally processed and acquired, and quantitative measurement and analysis of the turning response were then performed.

Fluorescent staining. Xenopus neurons were rapidly fixed with $4 \%$ paraformaldehyde and $0.25 \%$ glutaraldehyde in a cacodylate buffer $(0.1$ M sodium cacodylate and sucrose, $\mathrm{pH}$ 7.4) for $30 \mathrm{~min}$ and permeabilized with $0.1 \%$ Triton X-100 in Ringer's solution. Microtubules were labeled with a rat monoclonal antibody against $\beta$-tubulin (Harlan Bioproducts for Science, Indianapolis, IN) and a rabbit anti-rat IgG second antibody conjugated with FITC (Sigma, St. Louis, MO); the same cells were then labeled with $1 \%$ rhodamine phalloidin (Molecular Probes, Eugene, OR) for the actin filaments.

Live fluorescent imaging of MTs. Rhodamine-conjugated $\beta$-tubulin proteins (Cytoskeleton, Denver, $\mathrm{CO}$ ) were injected into one blastmere of Xenopus embryos at the two cell stage according to the method described previously (Chang et al., 1998). Neurons cultured from these embryos were examined by fluorescence microscopy using a $100 \times 1.3$ NA objective. A cooled CCD camera (PXL1400; Roper Scientifics, Tucson, AZ) was used for image acquisition, and the exposure time for each frame was $500 \mathrm{msec}$. To focally apply taxol, a micropipette ( $1 \mu \mathrm{m}$ opening $)$ containing taxol solution was placed at $50 \mu \mathrm{m}, 45^{\circ}$ away from the growth cone. Four to five images of the MTs of the growth cone were collected before the onset of the taxol application. Because of photo bleaching and toxicity from the high-resolution fluorescent imaging, we were only able to collect $\leq 20$ frames at different times after the onset of focal taxol application. Axon Imaging Workbench (Axon Instruments, Foster City, CA) was used for imaging and recording.

\section{RESULTS \\ Growth cone turning in diffusible guidance gradients requires dynamic microtubule ends}

To investigate the role of microtubules in growth cone steering, embryonic Xenopus spinal neurons grown on coverslips coated with Con A (Chang et al., 1998) were used 6-10 hr after plating (hereafter referred to as $6 \mathrm{hr}$ cultures) or 18-24 hr after plating (hereafter referred to as $18 \mathrm{hr}$ cultures). Xenopus neurons on Con A exhibited large and rapid-extending growth cones that allow spatially restricted manipulation of microtubule dynamics. Over a period of $\sim 30 \mathrm{~min}$, the average lengths of growth cone extension were $\sim 15$ and $20 \mu \mathrm{m}$ for 6 and $18 \mathrm{hr}$ cultures, respectively. We first examined the turning responses of the growth cones from 6 hr cultures to gradients of glutamate (Zheng et al., 1996a) and netrin-1 (Kennedy et al., 1994). A microscopic gradient of these molecules was created by repetitive pulsatile ejection of the solution from a micropipette placed $100 \mu \mathrm{m}$ away from the growth cone at a $45^{\circ}$ angle with respect to the original direction of extension (Lohof et al., 1992; Zheng et al., 1996a). Digital images of each growth cone at the onset and end of the 30 min gradient application were collected and overlaid to determine the response of the growth cone. Similar to our previous observation (Zheng et al., 1996a), a gradient of glutamate (50 $\mu \mathrm{M}$ in pipette) consistently induced marked attractive turning responses of Xenopus growth cones toward the glutamate source within $30 \mathrm{~min}$ in $6 \mathrm{hr}$ cultures (Fig. 1b), whereas the control group of growth cones showed no preferential response to the pipette application of culture medium (Fig. 1a). The average turning angle (in degrees) in the control group was $1.3 \pm 3.6 \mathrm{SEM}$, whereas that of growth cones exposed to glutamate was $9.4 \pm 2.9(p<0.05$; Kolmogorov-Smirnov test). The slightly smaller average turning angle than that observed previously for glutamate (Zheng et al., 1996a) likely resulted from the strong adhesion of these growth cones on the Con A-coated surface, which could limit the degree of steering.

We then tested the turning responses of Xenopus growth cones to a gradient of the guidance molecule netrin-1 (generously provided by Dr. M. Tessier-Lavigne, Stanford University, Stanford, CA) (Kennedy et al., 1994). In 6 hr cultures, a gradient of netrin-1 induced clear repulsion of the growth cones (Fig. 1d), resulting in an average turning angle of $-10.1 \pm 2(p<0.01$ compared with the $6 \mathrm{hr}$ control; Kolmogorov-Smirnov test). In 18 hr cultures, however, the same gradient of netrin-1 induced significant attraction of the growth cones (Fig. 1f). The average turning angle of $9.3 \pm 3.4$ was significantly different from $-0.2 \pm$ 3.3 of the $18 \mathrm{hr}$ control ( $p<0.05$; Kolmogorov-Smirnov test) (Table 1). This developmental switching of turning responses to netrin-1 has been observed previously in Xenopus growth cones grown on coverslips without any substrate coating (Ming et al., 2001) and may be associated with a change of intracellular levels of cAMP in these neurons (Wang and Zheng, 1998; Ming et al., 2001). To better depict the overall responses of a population of growth cones to these guidance gradients, cumulative histograms 

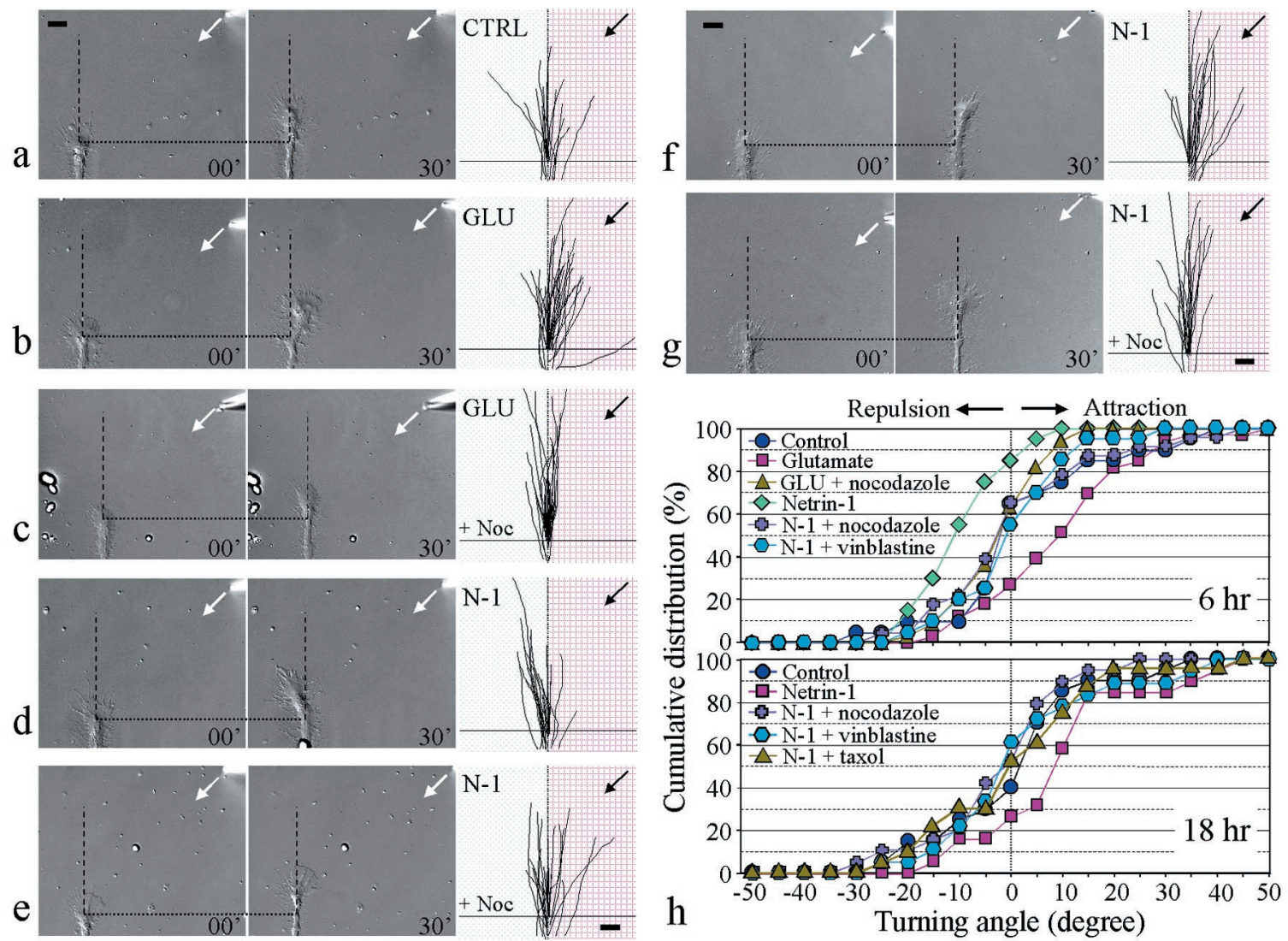

Figure 1. Blocking of growth cone turning responses to guidance gradients by inhibition of MT dynamics. $a-e$, Growth cone turning induced in 6 hr cultures. A gradient of glutamate $(b)$ or netrin-1 $(d)$ was applied to the growth cone by repetitive pulsatile pressure ejection of either $50 \mu \mathrm{M}$ glutamate $(G L U)$ or $5 \mu \mathrm{g} / \mathrm{ml}$ netrin-1 ( $N-1)$ solution from a micropipette (see Materials and Methods). The control ( $a$ ) was done with the same pulsatile pipette application of culture medium $(C T R L)$ to the growth cone. Images of the growth cone were collected at the onset (left image) and at the end (right image) of a $30 \mathrm{~min}$ application period. The origin is the center of the growth cone at the onset of the experiment. Dotted lines indicate corresponding positions along the neurite; dashed lines indicate the original direction of extension. Superimposed traces on the right (same magnifications as the growth cone images) depict the trajectory of neurite extension during the $30 \mathrm{~min}$ period for all of the growth cones in each group. Arrows indicate the direction of the gradient. Bath application of $10 \mathrm{~nm}$ nocodazole $(+N o c)$ abolished both attraction induced by glutamate $(c)$ and repulsion induced by netrin-1 $(e)$. $f, g$, Netrin-1-induced growth cone attraction in $18 \mathrm{hr}$ cultures $(f)$ was also blocked by the presence of 10 nm nocodazole in the bath $(g)$. Scale bars: $a-g$, $10 \mu \mathrm{m}$. $h$, The distribution of turning angles is presented to depict the overall responses under different experimental conditions. For each condition, the percentage value refers to the percentage of growth cones with the turning angle less than or equal to a given angular value. Data shown are turning responses induced by glutamate and netrin-1 in $6 \mathrm{hr}$ (top panel) and $18 \mathrm{hr}$ (bottom panel) cultures without and with the presence of the MT drugs nocodazole and vinblastine in bath.

of the distribution of turning angles (Fig. 1h) as well as the turning scores [percentages of growth cones scored as turning positively $(+)$, negatively ( - ), and having no turning response (0)] (Table 1) are presented. As shown clearly, a gradient of glutamate induced significant attraction, whereas a gradient of netrin-1 induced clear repulsion in $6 \mathrm{hr}$ cultures. For $18 \mathrm{hr}$ cultures, the majority of the growth cones turned positively toward the netrin-1 source. These turning results indicate that Con A coating did not alter the responsiveness of cultured Xenopus neurons to these guidance cues. Moreover, the fact that no significant change was observed in the extension rate for these conditions (Table 1) indicates that gradients of glutamate and netrin-1 exerted their effects primarily on the direction of growth cone extension.

To determine the role of MTs in growth cone turning responses induced by these diffusible gradients, we bath applied low concentrations of membrane-permeant MT-specific drugs to inhibit the dynamic MTs in the growth cone without causing significant MT depolymerization in the cell (Tanaka et al., 1995; Rochlin et al., 1996; Challacombe et al., 1997). The presence of a very low concentration $(10 \mathrm{~nm})$ of nocodazole in the bath did not significantly affect the extension rate of Xenopus growth cones but completely blocked both attraction induced by glutamate (Fig. 1c) and repulsion induced by netrin-1 (Fig. 1e) in $6 \mathrm{hr}$ cultures. The average turning angles induced by glutamate and netrin- 1 gradients in the presence of $10 \mathrm{~nm}$ nocodazole in bath were found to be $-2.5 \pm 1.4$ and $0.7 \pm 3.2$, respectively (Table 1 ). Statistical analyses using a Kolmogorov-Smirnov test indicated no preferential turning response induced by either gradient $(p>0.5$ compared with the $6 \mathrm{hr}$ control group; $p<0.01$ compared with their corresponding groups without nocodazole in bath). Similarly, growth cone attraction induced by netrin- 1 gradients in 18 hr cultures was also abolished by $10 \mathrm{~nm}$ nocodazole in bath (Fig. $1 g)$. The average turning angle of $-2.7 \pm 2.9$ was not different from that of the $18 \mathrm{hr}$ control ( $p>0.5$; Kolmogorov-Smirnov test). The complete elimination of both attractive and repulsive turning responses is better demonstrated by the cumulative histogram of the distribution of turning angles (Fig. 1h) and the turning scores (Table 1). Similar abolition of turning responses was also observed with a different MT-disrupting drug, vinblas- 
Table 1. Dynamic microtubule ends are required for growth cone turning induced by diffusible molecules

\begin{tabular}{|c|c|c|c|c|c|c|c|c|c|}
\hline \multirow[b]{2}{*}{ Chemicals in pipette } & \multirow[b]{2}{*}{ Chemicals in bath } & \multirow{2}{*}{$\begin{array}{l}\text { Pipette distance } \\
(\mu \mathrm{m})\end{array}$} & \multirow{2}{*}{$\begin{array}{l}\text { Culture } \\
\text { types }\end{array}$} & \multirow{2}{*}{$\begin{array}{l}\text { Turning angle } \\
\text { (degree)* }\end{array}$} & \multirow{2}{*}{$\begin{array}{l}\text { Extension } \\
(\mu \mathrm{m})^{*}\end{array}$} & \multicolumn{3}{|c|}{$\begin{array}{l}\text { Turning scores } \\
(\%)^{a}\end{array}$} & \multirow{2}{*}{$\begin{array}{l}\text { Number } \\
\text { of cells } \\
\text { examined }\end{array}$} \\
\hline & & & & & & + & 0 & - & \\
\hline None & None & 100 & $6 \mathrm{hr}$ & $1.3 \pm 3.6$ & $13.5 \pm 1.1$ & 30 & 45 & 25 & 20 \\
\hline $50 \mu \mathrm{M}$ glutamate & None & 100 & $6 \mathrm{hr}$ & $9.4 \pm 2.9^{* *}$ & $17.4 \pm 1.3$ & 61 & 21 & 18 & 33 \\
\hline $50 \mu \mathrm{M}$ glutamate & $10 \mathrm{~nm}$ nocodazole & 100 & $6 \mathrm{hr}$ & $2.5 \pm 1.4$ & $15.3 \pm 1.9$ & 18 & 46 & 36 & 33 \\
\hline $50 \mu \mathrm{M}$ glutamate & $7 \mathrm{~nm}$ taxol & 100 & $6 \mathrm{hr}$ & $-4.2 \pm 3.1$ & $10.4 \pm 0.8^{* *}$ & 25 & 40 & 35 & 20 \\
\hline $5 \mu \mathrm{g} / \mathrm{ml}$ netrin- $1^{b}$ & None & 100 & $6 \mathrm{hr}$ & $-10.1 \pm 2.0^{* *}$ & $15.1 \pm 1.6$ & 5 & 20 & 75 & 20 \\
\hline $5 \mu \mathrm{g} / \mathrm{ml}$ netrin $-1^{\mathrm{b}}$ & $10 \mathrm{~nm}$ nocodazole & 100 & $6 \mathrm{hr}$ & $0.7 \pm 3.2$ & $13.8 \pm 1.1$ & 30 & 40 & 30 & 23 \\
\hline $5 \mu \mathrm{g} / \mathrm{ml}$ netrin- $1^{b}$ & $4 \mathrm{~nm}$ vinblastine & 100 & $6 \mathrm{hr}$ & $0.2 \pm 2.6$ & $10.0 \pm 1.1^{* *}$ & 30 & 45 & 25 & 20 \\
\hline $5 \mu \mathrm{g} / \mathrm{ml}$ netrin $-1^{b}$ & $7 \mathrm{~nm}$ taxol & 100 & $6 \mathrm{hr}$ & $0.5 \pm 3.6$ & $12.1 \pm 2.0$ & 39 & 33 & 28 & 18 \\
\hline None & None & 100 & $18 \mathrm{hr}$ & $-0.2 \pm 3.3$ & $22.9 \pm 2.1$ & 30 & 40 & 30 & 20 \\
\hline $5 \mu \mathrm{g} / \mathrm{ml}$ netrin- 1 & None & 100 & $18 \mathrm{hr}$ & $9.3 \pm 3.4^{* *}$ & $19.2 \pm 1.5$ & 68 & 16 & 16 & 19 \\
\hline $5 \mu \mathrm{g} / \mathrm{ml}$ netrin- 1 & $10 \mathrm{~nm}$ nocodazole & 100 & $18 \mathrm{hr}$ & $-2.7 \pm 2.9$ & $20.2 \pm 1.8$ & 21 & 37 & 42 & 19 \\
\hline $5 \mu \mathrm{g} / \mathrm{ml}$ netrin- 1 & 4 nM vinblastine & 100 & $18 \mathrm{hr}$ & $1.2 \pm 3.6$ & $10.2 \pm 1.2^{* *}$ & 28 & 39 & 33 & 18 \\
\hline $5 \mu \mathrm{g} / \mathrm{ml}$ netrin- 1 & $7 \mathrm{~nm}$ taxol & 100 & $18 \mathrm{hr}$ & $1.1 \pm 3.2$ & $13.5 \pm 1.7^{* *}$ & 39 & 30 & 31 & 23 \\
\hline
\end{tabular}

*Values represent mean \pm SEM. **Significantly different from the control groups $(p<0.05$; Kolmogorov-Smirnov test).

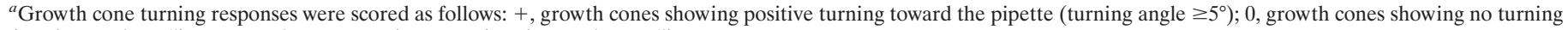
(turning angle $<5^{\circ}$ ); - , growth cones turning away (turning angle $\leq-5^{\circ}$ ).

${ }^{b}$ Growth cone repulsion induced by netrin-1 gradients was performed on neurons in $6 \mathrm{hr}$ cultures without NT-3 supplemented in the culture medium.

tine. Vinblastine binds to a different site on microtubules than nocodazole (Downing, 2000) and has been shown to inhibit the dynamic instability of single MTs in the growth cone without causing drastic MT depolymerization at low concentrations (Tanaka et al., 1995; Challacombe et al., 1997). Bath application of $4 \mathrm{nM}$ vinblastine completely abolished netrin-1-induced growth cone repulsion in $6 \mathrm{hr}$ cultures and attraction in 18 cultures, although growth cone extension was partially inhibited (Fig. 1h, Table 1). To further confirm that dynamic MTs in the growth cone are crucial, we used the MT-stabilizing drug taxol. Taxol at low concentrations has been shown to inhibit the dynamic instability of MTs and restrict MT distribution to the C-region of the growth cone over time (Williamson et al., 1996; Challacombe et al., 1997). Bath application of taxol at $7 \mathrm{~nm}$ slightly inhibited growth cone extension but completely abolished both attractive and repulsive responses induced by glutamate and netrin-1 gradients, respectively, in $6 \mathrm{hr}$ cultures (Table 1). Similarly, netrin-1-induced attraction in $18 \mathrm{hr}$ cultures was also blocked (Fig. 1h, Table 1).

Double fluorescent staining of MTs and the actin cytoskeleton was performed to confirm that these low concentrations of MT drugs specifically affected dynamic MTs in the P-region of the growth cone. Growth cones in control groups showed a typical pattern of these two cytoskeletal structures similar to that observed in other types of nerve growth cones (Smith, 1988). MT bundles were concentrated in the neurite shaft and primarily terminated in the $\mathrm{C}$-region of the growth cone, with a number of single MTs splaying into the P-region (Fig. 2a). These free MT ends have been shown to undergo rapid growth and shrinkage (dynamic instability) (Tanaka and Kirschner, 1991; Tanaka et al., 1995). Bath application of $7 \mathrm{~nm}$ taxol only for 20 min caused the withdrawal of many of these dynamic MTs from the P-region of the growth cone without apparent effect on the overall MT organization in the C-region (Fig. $2 b$ ); the actin cytoskeleton was not altered by the low concentration of taxol (Fig. 2b). For MT-disrupting drugs, both $10 \mathrm{~nm}$ nocodazole (Fig. $2 c$ ) and $4 \mathrm{~nm}$ vinblastine (Fig. $2 d$ ) did not affect the filamentous structures of the actin cytoskeleton in the growth cone, and they did not significantly disrupt MTs in the C-region of the growth cone and along the neurite. However, both drugs were found to primarily remove dynamic MT ends in the P-region of the growth cone, with vinblastine being most effective (Fig. $2 c, d$ ). Live fluorescent imaging of MTs also revealed that dynamic MT ends withdrew from the P-region of the growth cone shortly after the addition of these MT-disrupting drugs (data not shown). These results suggest that all three MT-specific drugs, at low concentrations, blocked the turning responses to guidance gradients by inhibiting the dynamic ends of MTs in the P-region of the growth cone. Together, our findings demonstrate that dynamic microtubule ends in the growth cone, particularly in the P-region, are essential for directional steering of the growth cone in response to extracellular guidance cues.

\section{Direct local modification of MT dynamics in the growth cone is sufficient to induce steering}

Dynamic MTs in the P-region of the growth cone are thought to play an exploratory role, and preferential stabilization and growth are believed to be involved in growth cone steering. To determine how local microtubule dynamics in the growth cone contribute to growth cone steering, we took advantage of the FLIP method (Zheng, 2000) to directly stabilize MTs in a spatial and temporal manner in the P-region of the growth cone where free MT ends were frequently observed (Fig. $3 a$ ). All subsequent experiments were performed in $6 \mathrm{hr}$ cultures. Neurons were incubated with the membrane-permeant photosensitive caged taxol [1 $\mu \mathrm{M}$ paclitaxel, 2'-(4,5-dimethoxy-2-nitrobenzyl)carbonate; Molecular Probes] for $10 \mathrm{~min}$ before focal photoactivation. FLIP of caged taxol in the growth cone was performed in a small spot of $\sim 2 \mu \mathrm{m}$ in diameter on one side of the growth cone (Fig. $3 a-c$, circles) and repeated at a rate of one pulse every $3 \mathrm{sec}$ (Zheng, 2000). A majority of the growth cones (18 of 23) exposed to $30 \mathrm{~min}$ of repetitive FLIP of caged taxol grew and steered toward the side of focal taxol release (Fig. $3 c, f$ ). Growth cones in the two control groups, one with laser irradiation but without caged taxol (Fig. $3 b, d)$ and the other without laser but with caged taxol (Fig. $3 e$ ), exhibited random turning responses. Comparisons of the turning 

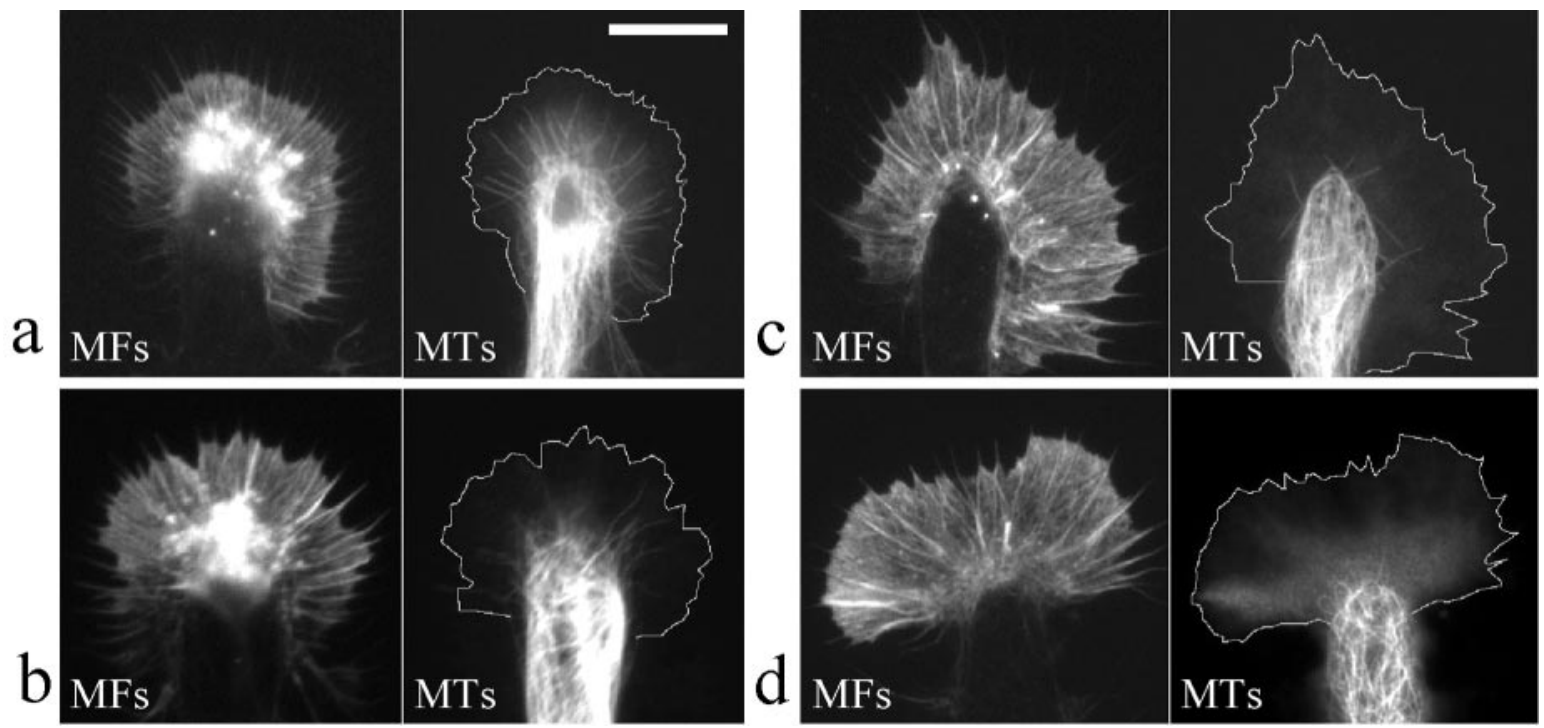

Figure 2. Double fluorescent imaging of microtubules and actin cytoskeleton in growth cones exposed to different MT-specific drugs. $a$, Representative pair of fluorescent images of the actin microfilaments $(M F s)$ and MTs of a control growth cone treated with medium only. $b-d$, Representative pairs of MT and MF images of Xenopus growth cones treated with (in nM) 7 taxol $(b), 10$ nocodazole $(c)$, and 4 vinblastine $(d)$. All of the cells were fixed at 20 min after the treatment. For clarity, the margin of the growth cone lamellipodia in MT staining has been outlined. Scale bar, $10 \mu \mathrm{m}$.
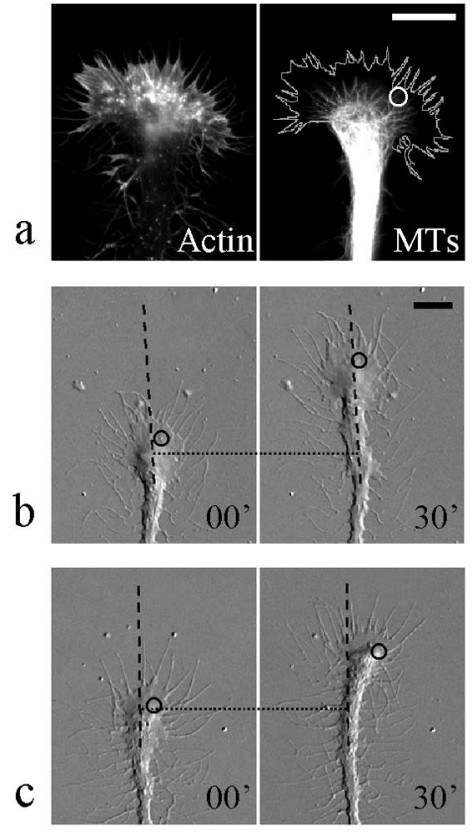
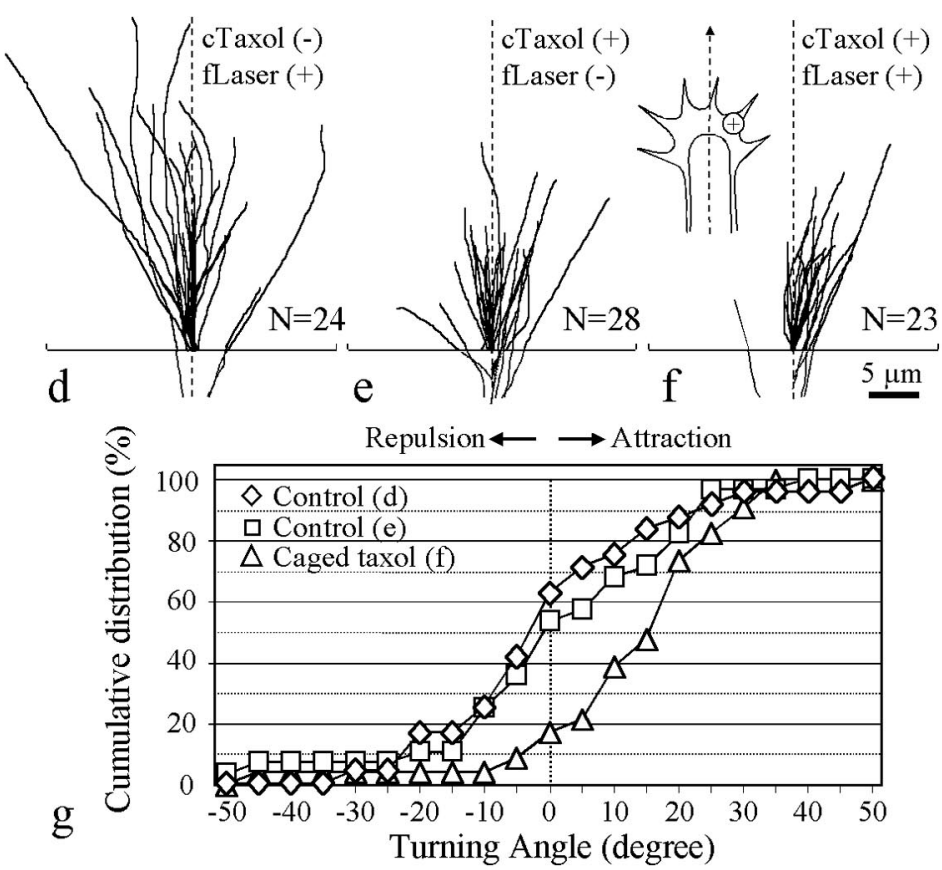

Figure 3. Attractive turning of Xenopus growth cones induced by repetitive FLIP of caged taxol. $a$, Immunostaining of the actin cytoskeleton and microtubules in the growth cone. For clarity, the margin of the growth cone lamellipodia in MT staining has been outlined. The circle indicates the location of the laser irradiation. $b$, Control growth cone (no caged taxol) at the beginning and end of 30 min of repetitive laser irradiation; circles indicate the laser spot. The dotted line indicates corresponding positions along the neurite; dashed lines indicate the original direction of extension. $c$, Growth cone loaded with caged taxol at the onset and end of $30 \mathrm{~min}$ of repetitive FLIP. Numbers in $b$ and $c$ represent minutes after the onset of repetitive laser irradiation. $d-f$, Superimposed traces of growth cone extension during the 30 min experimental period in two control groups [ $d$, focal laser $(f L a s e r)$ on but without caged taxol (cTaxol); $e$, laser off but with caged taxol] and in the group exposed to repetitive FLIP of caged taxol $(f)$. $g$, The cumulative distribution illustrates the overall turning responses of all of the growth cones examined in these three groups. Scale bars: $a-c, 10 \mu \mathrm{m} ; f, 5 \mu \mathrm{m}$.

angles of the growth cones exposed to FLIP of caged taxol and those of the two control groups showed that local release of caged taxol induced significant turning of the growth cones toward the side of taxol release (average turning angles: $11.7 \pm 3.5,-0.3 \pm$ 3.6 , and $-0.4 \pm 3.9$, respectively; $p<0.01$; Kolmogorov-Smirnov test) (Table 2). The cumulative histogram of the distribution of turning angles and the turning scores of these three groups (Fig. $3 g$, Table 2) clearly show that a greater number of growth cones exposed to FLIP of caged taxol turned with a positive angle than that of the two control groups. It should be noted that caged taxol appeared to exert some inhibition on neurite extension. The average length of neurite extension for growth cones exposed to 
Table 2. Growth cone attraction induced by FLIP of caged taxol

\begin{tabular}{|c|c|c|c|c|c|c|c|c|c|}
\hline & \multirow{2}{*}{$\begin{array}{l}\text { Laser } \\
\text { irradiation }\end{array}$} & \multirow{2}{*}{$\begin{array}{l}\text { Chemicals in } \\
\text { bath }\end{array}$} & \multirow[b]{2}{*}{ Culture types } & \multirow{2}{*}{$\begin{array}{l}\text { Turing angle } \\
\text { (degree)* }\end{array}$} & \multirow{2}{*}{$\begin{array}{l}\text { Extension } \\
(\mu \mathrm{m})^{*}\end{array}$} & \multicolumn{3}{|c|}{$\begin{array}{l}\text { Turning scores } \\
(\%)^{a}\end{array}$} & \multirow{2}{*}{$\begin{array}{l}\text { Number } \\
\text { of cells } \\
\text { examined }\end{array}$} \\
\hline & & & & & & + & 0 & - & \\
\hline Control 1 & + & None & $6 \mathrm{hr}$ & $-0.3 \pm 3.6$ & $19.1 \pm 1.6$ & 29 & 42 & 29 & 24 \\
\hline Control 2 & - & Caged taxol & $6 \mathrm{hr}$ & $0.4 \pm 3.9$ & $10.2 \pm 0.7^{* *}$ & 42 & 21 & 36 & 28 \\
\hline Uncaging & + & Caged taxol & $6 \mathrm{hr}$ & $11.7 \pm 3.5^{* *}$ & $10.2 \pm 0.8^{* *}$ & 78 & 13 & 9 & 23 \\
\hline
\end{tabular}

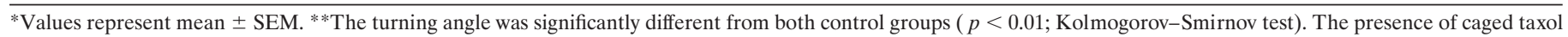
in bath appeared to inhibit neurite extension ( $p<0.01$ when comparing with control 1$)$.

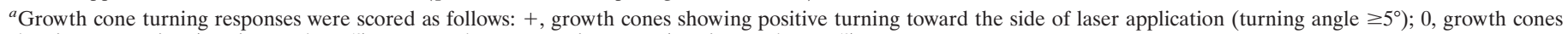
showing no turning (turning angle $<5^{\circ}$ ); - , growth cones turning away (turning angle $\leq-5^{\circ}$ ).

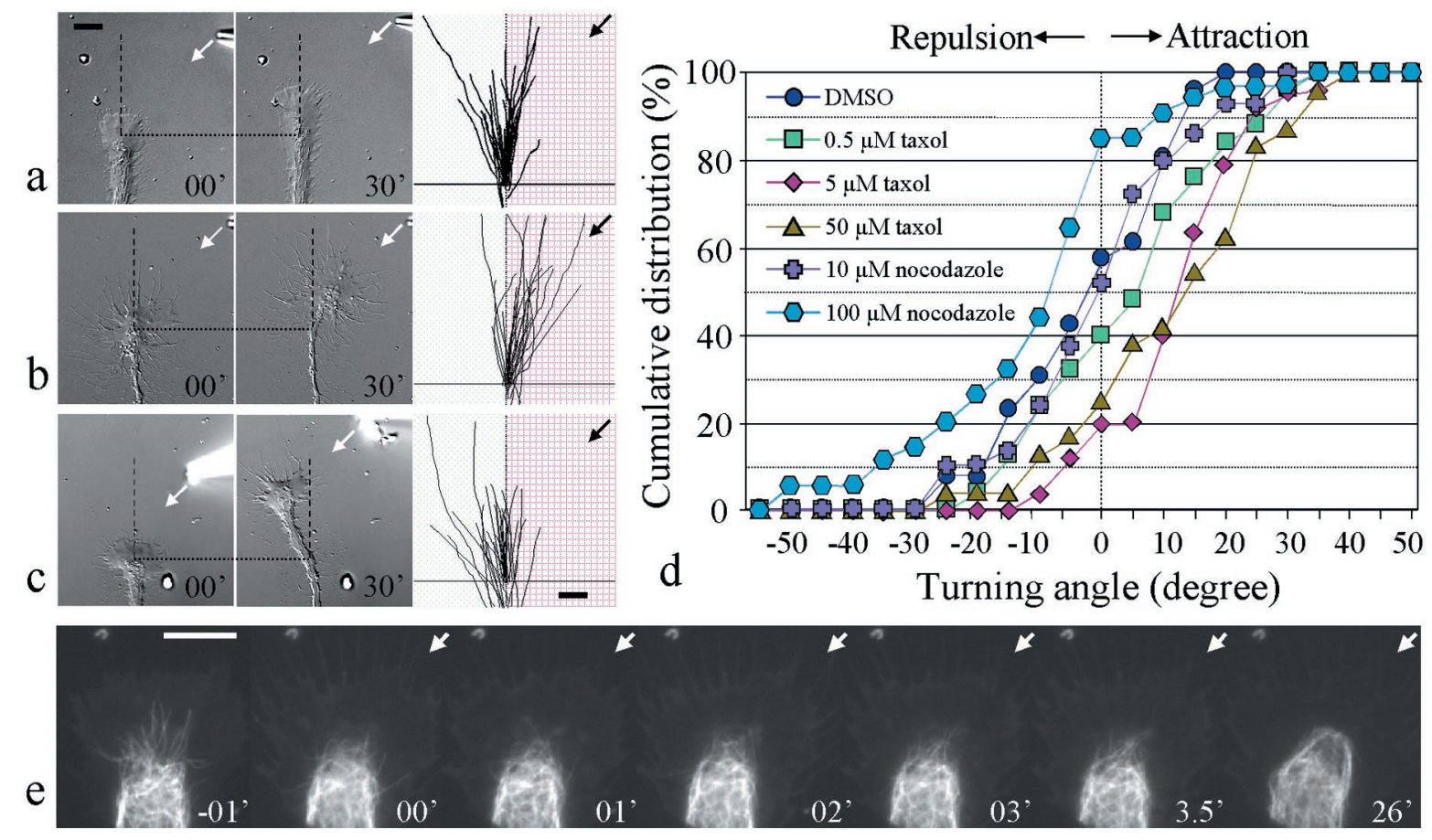

Figure 4. Turning responses induced by focal application of MT-specific drugs through a micropipette. $a$, In the control, growth cones were exposed to focal application of $1 \%$ DMSO. Representative images of a control growth cone at the onset (left image) and at the end (right image) of the 30 min application are shown together with the superimposed traces of all of the growth cones (same magnifications as the growth cone images). See the legend to Figure 1 for details. $b$, Attractive turning induced by focal application of $5 \mu \mathrm{M}$ taxol. $c$, Repulsive turning induced by focal application of $100 \mu \mathrm{M}$ nocodazole. Arrows in $a-c$ indicate the direction of the gradient. $d$, Overall responses of Xenopus growth cones to focal application of taxol and nocodazole are shown in the cumulative distribution of turning angles. $e$, Live imaging sequence of rhodamine-labeled MTs in a growth cone exposed to focal taxol application. Arrows indicate the direction of the focal taxol application. Numbers represent times after the onset of taxol application, with the first frame acquired 1 min before the onset of taxol application. Scale bars, $10 \mu \mathrm{m}$.

laser irradiation but without caged taxol was $19.1 \pm 1.6 \mu \mathrm{m}$, whereas the average lengths for growth cones in the presence of caged taxol with and without FLIP were $10.2 \pm 0.8$ and $10.3 \pm$ 0.7 , respectively (Table 2 ). This reduced extension may be attributable to some residual activity of caged taxol or a small amount of uncaging in response to ambient light. Nevertheless, spatially restricted application of taxol to one side of the growth cone clearly caused the growth cone to turn positively, thus demonstrating that local stabilization and possibly local promotion of MT polymerization of MTs are sufficient to induce a positive steering event (attractive turning).

To confirm the above finding, we examined whether focal extracellular application of the membrane-permeant taxol can also induce the growth cone to turn positively. We modified the pulsatile ejection method used for guidance gradients (Lohof et al., 1992; Zheng et al., 1996a) by moving the pipette to a distance of $50 \mu \mathrm{m}$ from the growth cone and reducing the pulse duration from the standard 20 to $5 \mathrm{msec}$ to create a much greater concentration difference of taxol reaching the two sides of the growth cone (Lohof et al., 1992). No turning response was observed in the control group of growth cones exposed to focal application of 1\% DMSO (Fig. 4a). Among 30 growth cones examined, random turning was observed, resulting in an average turning angle of $-1.3 \pm 2.5$ (Table 3 ). This result indicates that this focal application method did not produce any mechanical artifacts. When 5 or $50 \mu \mathrm{M}$ taxol (paclitaxel; Molecular Probes) was applied through the pipette to these growth cones, marked turning responses toward the source of taxol were observed (Fig. 4b, Table 3). The average turning angles were $11 \pm 2.2$ and $11 \pm 3.2$ for 5 and $50 \mu \mathrm{M}$ taxol (in pipette), respectively, and were significantly different from the DMSO control $(p<0.01$ and $<0.05$, respectively; Kolmogorov-Smirnov test). Higher concentrations of taxol, 
Table 3. Local modification of MT dynamics by pipette application induces growth cone turning

\begin{tabular}{|c|c|c|c|c|c|c|c|c|c|}
\hline \multirow[b]{2}{*}{ Chemicals in pipette } & \multirow[b]{2}{*}{ Chemicals in bath } & \multirow{2}{*}{$\begin{array}{l}\text { Pipette distance } \\
(\mu \mathrm{m})\end{array}$} & \multirow{2}{*}{$\begin{array}{l}\text { Culture } \\
\text { types }\end{array}$} & \multirow{2}{*}{$\begin{array}{l}\text { Turning angle } \\
\text { (degree)* }\end{array}$} & \multirow{2}{*}{$\begin{array}{l}\text { Extension } \\
(\mu \mathrm{m})^{*}\end{array}$} & \multicolumn{3}{|c|}{$\begin{array}{l}\text { Turning } \\
\text { scores }(\%)^{a}\end{array}$} & \multirow{2}{*}{$\begin{array}{l}\text { Number } \\
\text { of cells } \\
\text { examined }\end{array}$} \\
\hline & & & & & & + & 0 & - & \\
\hline $1 \%$ DMSO & None & 50 & $6 \mathrm{hr}$ & $-1.3 \pm 2.5$ & $14.0 \pm 1.1$ & 37 & 26 & 37 & 30 \\
\hline $0.5 \mu \mathrm{M}$ taxol & None & 50 & $6 \mathrm{hr}$ & $3.8 \pm 3.0$ & $14.2 \pm 1.1$ & 52 & 16 & 32 & 25 \\
\hline $5 \mu \mathrm{M}$ taxol & None & 50 & $6 \mathrm{hr}$ & $11.0 \pm 2.2^{* *}$ & $17.3 \pm 1.8$ & 80 & 8 & 12 & 25 \\
\hline $50 \mu \mathrm{M}$ taxol & None & 50 & $6 \mathrm{hr}$ & $11.0 \pm 3.2^{* *}$ & $15.2 \pm 1.5$ & 62 & 21 & 17 & 24 \\
\hline $5 \mu \mathrm{M}$ taxol & $20 \mathrm{~nm}$ cytochalasin D & 50 & $6 \mathrm{hr}$ & $-0.1 \pm 4.4$ & $12.8 \pm 1.4$ & 41 & 24 & 35 & 17 \\
\hline $5 \mu \mathrm{M}$ taxol & $100 \mathrm{pg} / \mathrm{ml}$ toxin $\mathrm{B}$ & 50 & $6 \mathrm{hr}$ & $0.5 \pm 3.1$ & $8.8 \pm 3.5^{* *}$ & 44 & 22 & 34 & 18 \\
\hline $10 \mu \mathrm{M}$ nocodazole & None & 50 & $6 \mathrm{hr}$ & $-0.7 \pm 2.7$ & $12.3 \pm 0.8$ & 28 & 34 & 38 & 29 \\
\hline $100 \mu \mathrm{M}$ nocodazole & None & 50 & $6 \mathrm{hr}$ & $-11.4 \pm 3.0^{* *}$ & $10.4 \pm 0.9 * *$ & 14 & 25 & 61 & 36 \\
\hline
\end{tabular}

*Values represent mean \pm SEM. **Significantly different from the control groups $(p<0.05$; Kolmogorov-Smirnov test).

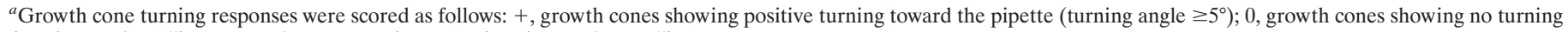
(turning angle $<5^{\circ}$ ); - , growth cones turning away (turning angle $\leq-5^{\circ}$ ).

however, were found to inhibit the growth cone extension, preventing further examination. When the taxol concentration was reduced to $0.5 \mu \mathrm{M}$, no significant turning was observed $(p>0.5$; Kolmogorov-Smirnov test). The cumulative histogram of the distribution of turning angles (Fig. $4 d$ ) and turning scores (Table 3 ) better depict the positive turning responses induced by focal taxol application. With the modified pipette application method, the concentration of taxol reaching the growth cone was estimated to be $\sim 1 / 400$ of the concentration in the pipette using the theoretical analysis described previously (Lohof et al., 1992). Therefore, the effective concentrations of taxol at the growth cone are $\sim 10-100 \mathrm{~nm}$ for inducing attraction. Live imaging of fluorescent MTs in the growth cone during taxol-induced turning showed that local taxol application caused preferential growth and bundling of MTs to the same side of the growth cone (Fig. $4 e$ ). These results, along with those from FLIP of caged taxol, clearly demonstrate that local stabilization and growth of MTs are sufficient to instructively induce attractive turning of the growth cones.

We then examined the effects of local destabilization and/or depolymerization of MTs on growth cone turning. Because none of the MT-disrupting drugs are available in caged form, we focally applied the membrane-permeant MT-disrupting drug nocodazole to one side of the growth cone through a micropipette. Focal application of $10 \mu \mathrm{M}$ nocodazole (in pipette) did not produce any effect on the direction and rate of growth cone extension (average turning angle, $-0.7 \pm 2.7 ; p>0.5$ compared with the DMSO control; Kolmogorov-Smirnov test) (Table 3). Focal application of $100 \mu \mathrm{M}$ nocodazole, however, caused the growth cones to steer away (Fig. 4c) (average turning angle, $-11.4 \pm 3 ; p<0.05$; Kolmogorov-Smirnov test). The cumulative histogram of the distribution of turning angles (Fig. 4d) and the turning scores (Table 3) also confirm that focal application of $100 \mu \mathrm{M}$ nocodazole (in pipette) caused a majority of the growth cones to steer away (repulsive turning). We were unable to test higher concentrations of nocodazole because of the inhibition of growth cone extension. Along with the results from focal taxol application, we have demonstrated that localized modification of MT dynamics on one side of the growth cone is sufficient to instruct the growth cone to steer in a specific direction. Local MT stabilization and growth initiate attractive turning, whereas local MT destabilization and disassembly induce repulsive turning.

\section{The actin cytoskeleton participates in MT-initiated growth cone attraction}

Although our results have clearly established an essential as well as instructive role for microtubules in directional steering of the growth cone, we have also found evidence for the involvement of the actin cytoskeleton in growth cone steering initiated by MTs. In growth cone attraction induced by focal taxol application, time-lapse DIC microscopy revealed preferential protrusion of lamellipodia on the side of the growth cone exposed to focal taxol application, before the actual turning of the growth cone (Fig. 5a, arrowheads). Because the specificity of taxol on MTs has been well documented (Diaz et al., 2000; Downing, 2000), this result suggests that local stabilization and growth of MTs can initiate the actin-based protrusive activity that may be important for directional steering of the growth cone. To test this idea and examine the role of the actin cytoskeleton in MT-initiated growth cone turning, we examined turning responses induced by taxol when actin polymerization was inhibited by a low concentration of cytochalasin D (CD). When $20 \mathrm{~nm} C D$ was present in the medium, the growth cone exhibited reduced actin-based motility as reviewed under high-resolution time-lapse DIC microscopy. Both retrograde membrane ruffling and the protrusion of lamellipodia and filopodia were partially inhibited. Furthermore, growth cone filopodia were lost over time by CD treatment (Zheng et al., 1996a) (see supplementary video clip at http:// www2.umdnj.edu/zhlabweb/video/CytoD.avi). However, considerable extension of the growth cone was still observed. We found that attractive turning of the growth cone induced by focal pipette application of $5 \mu \mathrm{M}$ taxol was completely abolished by the presence of $20 \mathrm{~nm} \mathrm{CD}$ in bath (Fig. 5b, Table 3), leading to an average turning angle of $-0.1 \pm 4.4(p>0.5$ compared with the DMSO control; $p<0.01$ compared with the $5 \mu \mathrm{M}$ taxol group; Kolmogorov-Smirnov test). The blockage of taxol-induced attraction is better depicted in the cumulative distribution of the turning angles (Fig. $5 d$ ). These results directly elucidate the involvement of the actin cytoskeleton in growth cone steering induced by localized MT activity.

Microtubules have been shown to regulate lamellipodial dynamics both in nerve growth cones (Tanaka and Kirschner, 1995; Gallo, 1998) and in migrating fibroblasts (Mikhailov and Gundersen, 1998) and to initiate actin-based intrapodia in motile growth cones (Rochlin et al., 1999). Taxol has been shown to induce rapid microtubule growth to subsequently produce lamel- 

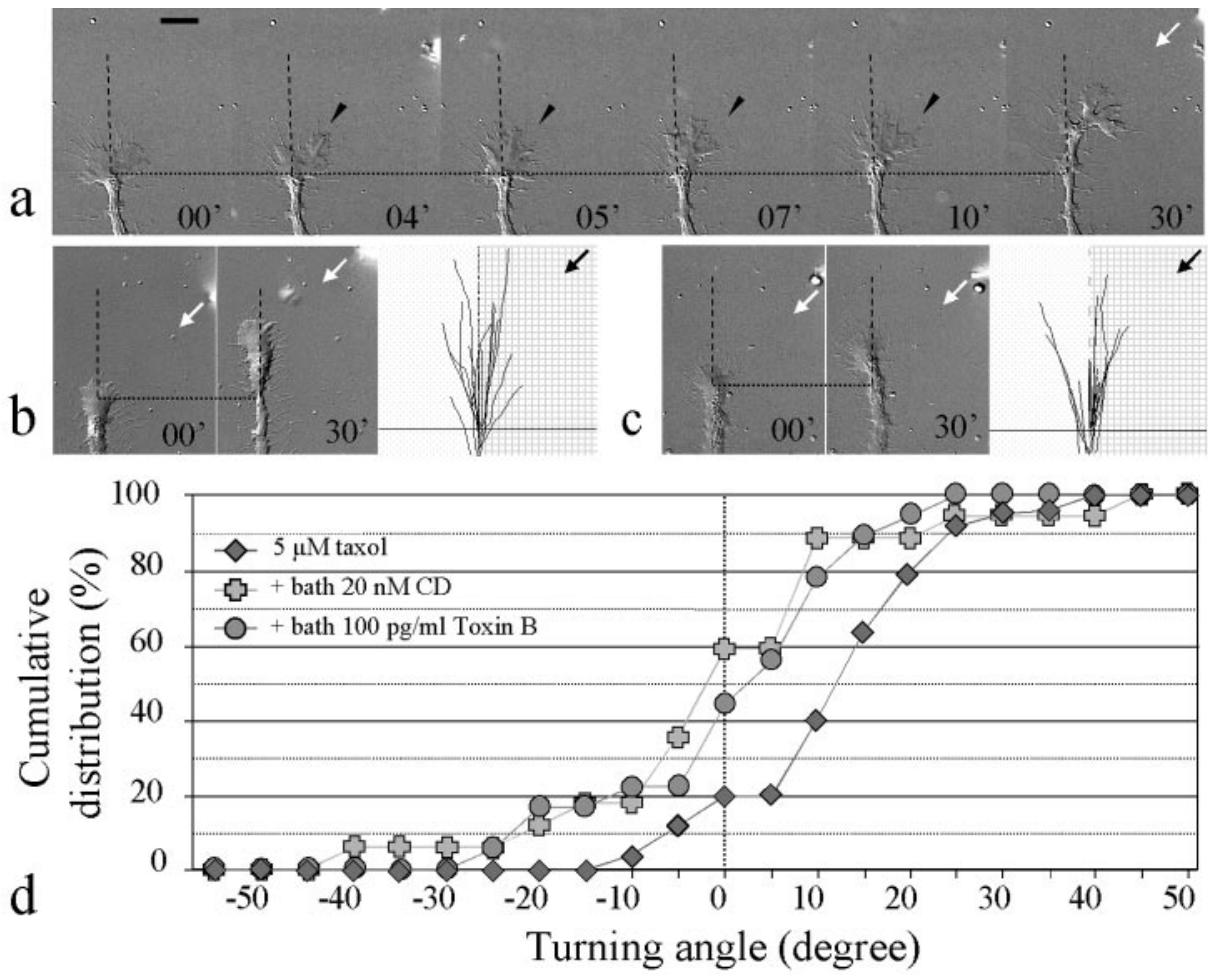

Figure 5. Involvement of the actin cytoskeleton in MT-initiated growth cone turning. $a$, Representative time lapse sequence of the attractive turning response of a Xenopus growth cone induced by focal taxol application. Note the increased protrusion of lamellipodia on the side of the growth cone facing the pipette before the actual turning of the growth cone (arrowheads). b, Growth cone response to focal application of $5 \mu \mathrm{M}$ taxol in the presence of 20 nM cytochalasin D in bath. Representative images of a growth cone at the onset (left image) and at the end (right image) of the $30 \mathrm{~min}$ taxol application are shown together with the superimposed traces of all of the growth cones on the right (same magnifications as the growth cone images). See the legend to Figure 1 for details. $c$, Growth cone response to focal application of $5 \mu \mathrm{M}$ taxol in the presence of $100 \mathrm{pg} / \mathrm{ml}$ toxin B in bath. Scale bar, $10 \mu \mathrm{m}$. Arrows indicate the direction of the taxol gradient. $d$, Overall responses of growth cones to focal taxol application in the presence of toxin B (to inhibit the Rho GTPases) and cytochalasin D (to inhibit the actin assembly) are shown as the cumulative distribution of turning angles. lipodial protrusion in migrating fibroblasts (Waterman-Storer et al., 1999). Recently, microtubule growth was found to activate Rac1, a member of the Rho GTPases (Rho, Rac, and Cdc-42) (Hall, 1998), to induce lamellipodial protrusion (WatermanStorer et al., 1999). To test this possibility, we bath applied toxin $\mathrm{B}$ (from Clostridium difficile; Cytoskeleton) to inactivate the Rho family of GTPases (Just et al., 1995). Toxin B at $100 \mathrm{pg} / \mathrm{ml}$ was found to completely abolish the attractive turning of growth cones induced by focal taxol application (Fig. $5 c$, Table 3), resulting in an average turning angle of $0.5 \pm 3.1(p>0.5$ compared with the DMSO control; $p<0.01$ compared with the $5 \mu \mathrm{M}$ group; Kolmogorov-Smirnov test). The blockage of taxol-induced attraction is also illustrated by the cumulative distribution of the turning angles of the entire population of growth cones examined, which is similar to that seen in the presence of CD (Fig. $5 d$ ); both $\mathrm{CD}$ and toxin $\mathrm{B}$ effectively blocked the attraction induced by local taxol application. However, unlike CD, toxin B exerted little effect on the actin-based motility of the growth cone. Both retrograde membrane ruffling and the protrusion of filopodia and lamellipodia were not affected over the time period of the recording, and the growth cone morphology was not significantly altered (see the supplementary time-lapse video clip at http://www2.umdnj.edu/ zhlabweb/video/ToxinB.avi). These observations suggest that toxin B did not block taxol-induced growth cone turning through inhibition of the actin dynamics. Rather, it is likely that toxin B blocked taxol-induced turning through its specific inhibition of Rho GTPases signaling. These results demonstrate that the MTdirected steering mechanism involves the actin cytoskeleton. Local stabilization and growth of MTs on one side of the growth cone is sufficient to initiate the steering event by inducing sitedirected, actin-based protrusion, possibly through the activation of the Rho GTPases (e.g., Rac1), to complete the turning. With the existence of molecules that are capable of interacting with both cytoskeletal networks (Vega and Solomon, 1997; WatermanStorer and Salmon, 1999), it is conceivable that coordinated efforts between the microtubules and actin cytoskeleton are fundamental for successful steering of the growth cone in specific directions.

\section{DISCUSSION}

In this study, we have presented three pieces of direct evidence to demonstrate an essential and instructive role of microtubules in growth cone steering. First, we show that both attraction and repulsion induced by diff usible guidance gradients can be blocked by inhibition of the MT dynamics through low concentrations of MT-specific drugs. We then demonstrate, to our knowledge for the first time, that local modification of the MT dynamics in the growth cone is sufficient to induce growth cone steering: local stabilization and growth induce growth cone attraction, and local destabilization causes growth cone repulsion. Finally, we show that the actin cytoskeleton actively participates in MT-initiated growth cone turning; local MT stabilization and growth likely activate the Rho family of GTPases to induce the actin-based protrusive activities to complete the turning process. These results indicate the existence of a novel steering mechanism in which microtubules play a leading role for growth cone turning.

The actin filaments and microtubules are two fundamental cytoskeletal components for growth cone motility (Mitchison and Kirschner, 1988). There is ample evidence to support a major role for the actin cytoskeleton in directional movement of nerve growth cones: (1) actin filaments are concentrated at the peripheral region of the growth cone that is tightly associated with motility (Smith, 1988; Lin and Forscher, 1995), (2) disruption of the actin-based filopodia and lamellipodia blocks growth cone turning induced by extracellular cues (Bentley and ToroianRaymond, 1986; Chien et al., 1993; Zheng et al., 1996a), and (3) families of actin-associated proteins have been identified and implicated in various signaling pathways to regulate the dynamics of the actin cytoskeleton (Hall, 1998; Pollard et al., 2000). The precise function of MTs and their associated proteins in growth 
cone motility, particularly in directional growth cone steering, has not been elucidated. Although neuronal processes are still capable of extension when the actin cytoskeleton is disrupted by cytochalasins (Marsh and Letourneau, 1984; Bradke and Dotti, 1999), it is generally thought that the major function of MTs is to consolidate and provide mechanical support to the new extension initiated by the actin cytoskeleton. Local actin-based motility initiates and leads the steering sequence that involves subsequent local stabilization of these dynamic MTs followed by selective MT growth (Mitchison and Kirschner, 1988; Bentley and O'Connor, 1994). Recently, however, an increasing body of evidence suggests that MTs may play a more active role in directional motility of the growth cone (Tanaka and Kirschner, 1995; Tanaka et al., 1995; Challacombe et al., 1997; Mack et al., 2000; Dent and Kalil, 2001). Dynamic microtubule ends have been observed to explore the actin-rich P-region of the growth cone, and inhibition of these dynamic MT ends in the growth cone was found to abolish repulsive turning at the substrate border (Tanaka and Kirschner, 1995; Williamson et al., 1996; Challacombe et al., 1997). In this study, we used low concentrations of MT-specific drugs to test the role of MTs in growth cone turning in response to diffusible cues. Low concentrations of MTstabilizing (taxol) or MT-destabilizing (nocodazole and vinblastine) drugs inhibit free dynamic microtubule ends in the growth cone P-region but do not inhibit axon extension, alter growth cone morphology, or grossly disrupt the cytoskeletal architecture. Both attractive and repulsive turning responses to guidance gradients were abolished by the presence of these drugs in the medium. These results have further substantiated the previous findings in growth cone repulsion at the substrate boundary and have established the fundamental importance of dynamic microtubule ends in both growth cone attraction and repulsion to diffusible guidance cues.

The most significant finding of this study is the demonstration of an instructive role of microtubules in growth cone turning. Through direct focal application of the MT-specific drugs taxol and nocodazole to one side of the growth cone, we show that local modification of MT dynamics in the growth cone is sufficient to induce directional steering of the growth cone. Both FLIP and pipette techniques allowed us to introduce MT-specific drugs in a spatially restricted manner within the growth cone. When MTs are locally stabilized, the growth cone turns toward the site of stabilization, whereas local destabilization results in turning away from the site of destabilization. It should be emphasized that both taxol and nocodazole are applied in a local and pulsatile manner either through photoactivation or through pipette application. These MT-specific drugs are thus spatially restricted to only a small region of the growth cone at low concentrations. For example, the peak taxol concentration reaching the growth cone is estimated to be $\sim 10 \mathrm{~nm}$ (corresponding to $5 \mu \mathrm{M}$ in pipette) or less on the application side but much lower on the distal side of the growth cone. Therefore, local and pulsatile application of these MT drugs is most likely to produce only local effects on MT dynamics without grossly inhibiting MT dynamics in the entire growth cone, thus allowing the growth and turning of the growth cone. Our live imaging of fluorescent MTs showed that local taxol application promoted MT growth to the side of taxol application. The effects of taxol certainly depend on its concentrations as well as its spatial distribution; a high concentration of taxol applied to the entire growth cone and the cell would likely result in the inhibition of growth cone extension. The live imaging of fluorescent MTs itself, however, appeared to exert some adverse effect on dynamic MTs in the P-region, which prevented detailed studies on the dynamics of MTs in the growth cone during turning induced by local MT drugs. Such studies would be important for a better understanding of how local MT dynamics regulate the growth cone steering. In particular, with an improved dualwavelength live-imaging method of both cytoskeletal systems (Salmon et al., 2002), one would be able to address how the local MT dynamics affect the local dynamics of the actin cytoskeleton.

Our turning results were obtained using spatially restricted MT-specific drugs. It is possible that intracellular signaling components downstream of guidance receptors use a similar strategy to promote MT stabilization followed by local polymerization in a region of the growth cone that will ultimately determine the direction of new extension. Conversely, the observation that depolymerization of the dynamic MTs on one side of the growth cone results in a turning response in the other direction indicates that an alternate strategy could be to inhibit local microtubule extension to promote a turn in the opposite direction. Although our pipette application method does not exclusively restrict the MT drugs to only one side of the growth cone, the turning responses to focal taxol and nocodazole application appeared to be as significant as those induced by guidance gradients of netrin-1 and glutamine. During guidance, spatially and temporally restricted signaling events could result in much more localized modification of MT dynamics to effectively steer the growth cone. It would be of great interest to examine whether and how some specific intracellular signals (e.g., $\mathrm{Ca}^{2+}$ ) influence local microtubule polymerization, depolymerization, or a combination of both during turning responses to guidance cues.

Finally, the finding that the actin cytoskeleton participates in MT-initiated growth cone turning reveals that the interplay between these two cytoskeletal systems is critically important for directional movement of the growth cone. Our conclusion is based on three experimental observations: (1) high-resolution, time-lapse imaging revealed preferential lamellipodial protrusion on the side of focal taxol application, (2) complete blockade of taxol-induced growth cone attraction by cytochalasin D, and (3) abolition of taxol-induced growth cone attraction by toxin $\mathrm{B}$, a toxin that specifically inactivates the Rho family small GTPases (Just et al., 1995). These findings are consistent with the emerging evidence on the importance of interaction between these two cytoskeletal systems in cell motility (Dent and Kalil, 2001; Salmon et al., 2002; Schaefer et al., 2002). Additionally, more recent findings directly demonstrate the interaction between microtubules and the Rho family small GTPases (Fukata et al., 2002; Krendel et al., 2002), further suggesting a role for Rho GTPases in microtubule and actin interaction. Together, our results indicate an MT-initiated steering mechanism that requires coordinated activities between these two cytoskeleton systems. The identification of signaling pathways that interact with and/or target MTs (Gundersen and Cook, 1999) provides the potential for signals from the extracellular environment to regulate MT dynamics to control the directional motility of growth cones. It is conceivable that different extracellular cues may act through different signaling pathways that could target the actin cytoskeleton and/or MTs independently, but the concerted efforts between these two systems are required to enable the growth cone to respond effectively to guidance cues. It is possible that signals elicited by extracellular cues may act either as primary signals that locally modify MTs as a first step in initiation or as reinforcing signals in a feedback loop downstream of actin. Our data demonstrate that at the very least, localized perturbations in micro- 
tubule dynamics are indeed sufficient to initiate and direct new growth cone extension. Thus, the next step is to identify and determine the role of some of the signaling molecules that target MT dynamics in growth cone guidance. Nevertheless, a two-way steering system in which either microtubules or the actin cytoskeleton can take the driver's seat clearly provides developing axons with advantages to effectively respond to a wide variety of guidance cues.

\section{REFERENCES}

Bentley D, O'Connor T (1994) Cytoskeletal events in growth cone steering. Curr Opin Neurobiol 4:43-48.

Bentley D, Toroian-Raymond A (1986) Disoriented pathfinding by pioneer neurone growth cones deprived of filopodia by cytochalasin treatment. Nature 323:712-715.

Bradke F, Dotti CG (1999) The role of local actin instability in axon formation. Science 283:1931-1934.

Challacombe JF, Snow DM, Letourneau PC (1997) Dynamic microtubule ends are required for growth cone turning to avoid an inhibitory guidance cue. J Neurosci 17:3085-3095.

Chang S, Rodionov VI, Borisy GG, Popov SV (1998) Transport and turnover of microtubules in frog neurons depend on the pattern of axonal growth. J Neurosci 18:821-829.

Chien CB, Rosenthal DE, Harris WA, Holt CE (1993) Navigational errors made by growth cones without filopodia in the embryonic $\mathrm{Xe}$ nopus brain. Neuron 11:237-251.

Dent EW, Kalil K (2001) Axon branching requires interactions between dynamic microtubules and actin filaments. J Neurosci 21:9757-9769.

Diaz JF, Strobe R, Engelborghs Y, Souto AA, Andreu JM (2000) Molecular recognition of taxol by microtubules. Kinetics and thermodynamics of binding of fluorescent taxol derivatives to an exposed site. J Biol Chem 275:26265-26276.

Downing KH (2000) Structural basis for the interaction of tubulin with proteins and drugs that affect microtubule dynamics. Annu Rev Cell Dev Biol 16:89-111.

Fukata M, Watanabe T, Noritake J, Nakagawa M, Yamaga M, Kuroda S, Matsuura Y, Iwamatsu A, Perez F, Kaibuchi K (2002) Rac1 and Cdc42 capture microtubules through IQGAP1 and CLIP-170. Cell 109:873-885

Gallo G (1998) Involvement of microtubules in the regulation of neuronal growth cone morphologic remodeling. J Neurobiol 35:121-140.

Gundersen GG, Cook TA (1999) Microtubules and signal transduction. Curr Opin Cell Biol 11:81-94.

Hall A (1998) Rho GTPases and the actin cytoskeleton. Science 279:509-514

Jay DG (1996) Molecular mechanisms of directed growth cone motility. Perspect Dev Neurobiol 4:137-145.

Just I, Selzer J, Wilm M, von Eichel-Streiber C, Mann M, Aktories K (1995) Glucosylation of Rho proteins by Clostridium difficile toxin B. Nature 375:500-503.

Kennedy TE, Serafini T, de la Torre JR, Tessier-Lavigne M (1994) Netrins are diff usible chemotropic factors for commissural axons in the embryonic spinal cord. Cell 78:425-435.

Krendel M, Zenke FT, Bokoch GM (2002) Nucleotide exchange factor GEF-H1 mediates cross-talk between microtubules and the actin cytoskeleton. Nat Cell Biol 4:294-301.

Lin CH, Forscher P (1995) Growth cone advance is inversely proportional to retrograde F-actin flow. Neuron 14:763-771.

Lin CH, Thompson CA, Forscher P (1994) Cytoskeletal reorganization underlying growth cone motility. Curr Opin Neurobiol 4:640-647.

Lohof AM, Quillan M, Dan Y, Poo MM (1992) Asymmetric modulation of cytosolic cAMP activity induces growth cone turning. J Neurosci $12: 1253-1261$.

Mack TG, Koester MP, Pollerberg GE (2000) The microtubuleassociated protein MAP1B is involved in local stabilization of turning growth cones. Mol Cell Neurosci 15:51-65.
Marsh L, Letourneau PC (1984) Growth of neurites without filopodial or lamellipodial activity in the presence of cytochalasin B. J Cell Biol 99:2041-2047.

Mikhailov A, Gundersen GG (1998) Relationship between microtubule dynamics and lamellipodium formation revealed by direct imaging of microtubules in cells treated with nocodazole or taxol. Cell Motil Cytoskeleton 41:325-340.

Ming G, Henley J, Tessier-Lavigne M, Song H, Poo M (2001) Electrical activity modulates growth cone guidance by diff usible factors. Neuron 29:441-452.

Mitchison T, Kirschner M (1988) Cytoskeletal dynamics and nerve growth. Neuron 1:761-772

Parent CA, Devreotes PN (1999) A cell's sense of direction. Science 284:765-770.

Pollard TD, Blanchoin L, Mullins RD (2000) Molecular mechanisms controlling actin filament dynamics in nonmuscle cells. Annu Rev Biophys Biomol Struct 29:545-576.

Rochlin MW, Wickline KM, Bridgman PC (1996) Microtubule stability decreases axon elongation but not axoplasm production. J Neurosci 16:3236-3246.

Rochlin MW, Dailey ME, Bridgman PC (1999) Polymerizing microtubules activate site-directed F-actin assembly in nerve growth cones. Mol Biol Cell 10:2309-2327.

Salmon WC, Adams MC, Waterman-Storer CM (2002) Dual-wavelength fluorescent speckle microscopy reveals coupling of microtubule and actin movements in migrating cells. J Cell Biol 158:31-37.

Schaefer AW, Kabir N, Forscher P (2002) Filopodia and actin arcs guide the assembly and transport of two populations of microtubules with unique dynamic parameters in neuronal growth cones. J Cell Biol 158:139-152.

Smith SJ (1988) Neuronal cytomechanics: the actin-based motility of growth cones. Science 242:708-715.

Spitzer NC, Lamborghini JE (1976) The development of the action potential mechanism of amphibian neurons isolated in culture. Proc Natl Acad Sci USA 73:1641-1645.

Suter DM, Forscher P (2000) Substrate-cytoskeletal coupling as a mechanism for the regulation of growth cone motility and guidance. J Neurobiol 44:97-113.

Tanaka EM, Kirschner MW (1991) Microtubule behavior in the growth cones of living neurons during axon elongation. J Cell Biol 115:345-363.

Tanaka E, Kirschner MW (1995) The role of microtubules in growth cone turning at substrate boundaries. J Cell Biol 128:127-137.

Tanaka E, Ho T, Kirschner MW (1995) The role of microtubule dynamics in growth cone motility and axonal growth. J Cell Biol 128:139-155.

Tessier-Lavigne M, Goodman CS (1996) The molecular biology of axon guidance. Science 274:1123-1133.

Vega LR, Solomon F (1997) Microtubule function in morphological differentiation: growth zones and growth cones. Cell 89:825-828.

Wang Q, Zheng JQ (1998) Cyclic AMP-mediated regulation of neurotrophin-induced collapse of nerve growth cones. J Neurosci 18:4973-4984.

Waterman-Storer CM, Salmon E (1999) Positive feedback interactions between microtubule and actin dynamics during cell motility. Curr Opin Cell Biol 11:61-67.

Waterman-Storer CM, Worthylake RA, Liu BP, Burridge K, Salmon ED (1999) Microtubule growth activates Rac1 to promote lamellipodial protrusion in fibroblasts. Nat Cell Biol 1:45-50.

Williamson T, Gordonweeks PR, Schachner M, Taylor J (1996) Microtubule reorganization is obligatory for growth cone turning. Proc Natl Acad Sci USA 93:15221-15226.

Zheng JQ (2000) Turning of nerve growth cones induced by localized increases in intracellular calcium ions. Nature 403:89-93.

Zheng JQ, Wan JJ, Poo MM (1996a) Essential role of filopodia in chemotropic turning of nerve growth cone induced by a glutamate gradient. J Neurosci 16:1140-1149.

Zheng JQ, Poo MM, Connor JA (1996b) Calcium and chemotropic turning of nerve growth cones. Perspect Dev Neurobiol 4:205-213. 